\title{
Biological Inspired Stochastic Optimization Technique (PSO) for DOA and Amplitude Estimation of Antenna Arrays Signal Processing in RADAR Communication System
}

\author{
Khurram Hammed, Sajjad Ahmed Ghauri, and M. Salman Qamar \\ Department of Electronics Engineering, International Islamic University, Islamabad, Pakistan \\ Correspondence should be addressed to Sajjad Ahmed Ghauri; sajjad.ghauri.101@gmail.com
}

Received 6 August 2015; Revised 27 October 2015; Accepted 28 October 2015

Academic Editor: Fanli Meng

Copyright (C) 2016 Khurram Hammed et al. This is an open access article distributed under the Creative Commons Attribution License, which permits unrestricted use, distribution, and reproduction in any medium, provided the original work is properly cited.

\begin{abstract}
This paper presents a stochastic global optimization technique known as Particle Swarm Optimization (PSO) for joint estimation of amplitude and direction of arrival of the targets in RADAR communication system. The proposed scheme is an excellent optimization methodology and a promising approach for solving the DOA problems in communication systems. Moreover, PSO is quite suitable for real time scenario and easy to implement in hardware. In this study, uniform linear array is used and targets are supposed to be in far field of the arrays. Formulation of the fitness function is based on mean square error and this function requires a single snapshot to obtain the best possible solution. To check the accuracy of the algorithm, all of the results are taken by varying the number of antenna elements and targets. Finally, these results are compared with existing heuristic techniques to show the accuracy of PSO.
\end{abstract}

\section{Introduction}

In RADAR communication system, it is very important apprehension to accurate estimation of direction of arrival and amplitude. Plenty of work has been done in this area by implementing classical and metaheuristic techniques. With the passage of time applicability of these schemes enhances drastically due to provisioning of better results in low signal to noise ratio. These techniques comprise Particle Swarm Optimization (PSO), Genetic Algorithm (GA), Ant Colony Optimization (ACO), and so forth. In this study, biological inspired method named PSO is applied for joint estimation of direction of arrival and amplitude for the targets located in far field of the antenna arrays. Results obtained from this scheme are compared with GA-PS and GA-Fmincon to understand the importance of PSO. Different cases are discussed by varying the number of targets in the air.

Moreover, this paper is organized as follows: Section 2 addresses the different local and global schemes related to DOA and amplitude estimation. Next section is comprised of the mathematical modeling on the RADAR antenna arrays system. Brief discussion on proposed methodology is summed up in Section 4. In Section 5 simulated results are shown and finally some conclusions and future recommendations by the authors are suggested in Section 6 .

\section{Related Work}

Today, an enormous research has been put through for accurate estimation of direction of arrival [1] in adaptive arrays signal processing [2] and communication systems. It has the vast applications in biomedical technology, RADARs [3], SONARs [4], and cellular wireless networks [5]. Smart antenna arrays play very promising role in unidentified time varying scenarios. So in this course of study we are primarily focused on the estimation of DOA and amplitude of the received signal from far field targets by using smart antenna uniform linear arrays in RADAR. Smart antenna system contains an array of radiating and receiving sensors. These 
arrays have the capability to steer the main beam in any desired direction in space, while placing suitable nulls in direction of unwanted signals or jammers [6]. Along with these features, smart antenna arrays system has one difficulty that we do not identify, the direction of arrival of the received signal. For that reason, it is necessary to estimate DOA of the received signal to design a really smart adaptive antenna array system.

To overcome this issue, there are various classical methods to estimate DOA such as Multiple Signal Classification (MUSIC) [7], Maximum Likelihood (ML) method [8, 9], Bartlett method [10], and Capon method [11]. But to fulfill the military requirements, we need to apply such algorithms that have high accuracy and quick response and provide better results in case of real time scenarios. By keeping in view these standards, one can implement biological inspired techniques such as global search algorithms [12] like Particle Swarm Optimization (PSO), Genetic Algorithm (GA) [13], Ant Colony Optimization (ACO) [14], and so forth and local search algorithms [15] like Interior Point Algorithm [16], Active Set Algorithm [17], Sequential Quadratic Programming [18], and so forth.

In this paper, we aim to estimate both amplitude and DOA in RADAR antenna arrays by applying Particle Swarm Optimization (PSO) technique. There are various methods to estimate DOA and amplitude in RADAR communication system as mentioned above but the beauty of PSO is that it provides the most precise, consistently convergent, and feasible results. Instead of other approaches PSO is very simple and easily completed and needs fewer parameters which makes it fully developed. Due to fewer parameters in the algorithm it takes very small executing time as compared to other popular techniques. PSO algorithm is derived from birds and fish flock movement behavior and it is one of the promising algorithms used to solve global optimization issues. It requires only primitive mathematical operators and is computationally inexpensive in terms of both memory requirements and speed [19]. That is why PSO is proposed to estimate DOA and amplitude in RADAR communication system in this paper.

\section{Problem Formulation}

In this portion of the paper, we formulate the problem for far field targets by using uniform linear array (ULA) system in radar receiver. ULA consist of $M$ number of antenna elements and all elements are equally distant as shown in Figure 1. Due to a variety of clutters in air, we received many numbers of signals at ULA. This makes our problem more complex; to avoid this complexity we assume that there are $L$ number of targets in far field of ULA and $L$ number of signals are impinging on RADAR antenna arrays. $M$ is always greater then $L$ for accurate and optimum solution.

All targets are assumed to be in narrow band and have known frequency $\left(\omega_{0}\right)$, where each target is occupying different direction of arrival $(\varphi)$ and amplitude $(\alpha)$. However, signal received at the reference antenna element has no phase shift but signal received at other elements will undergo

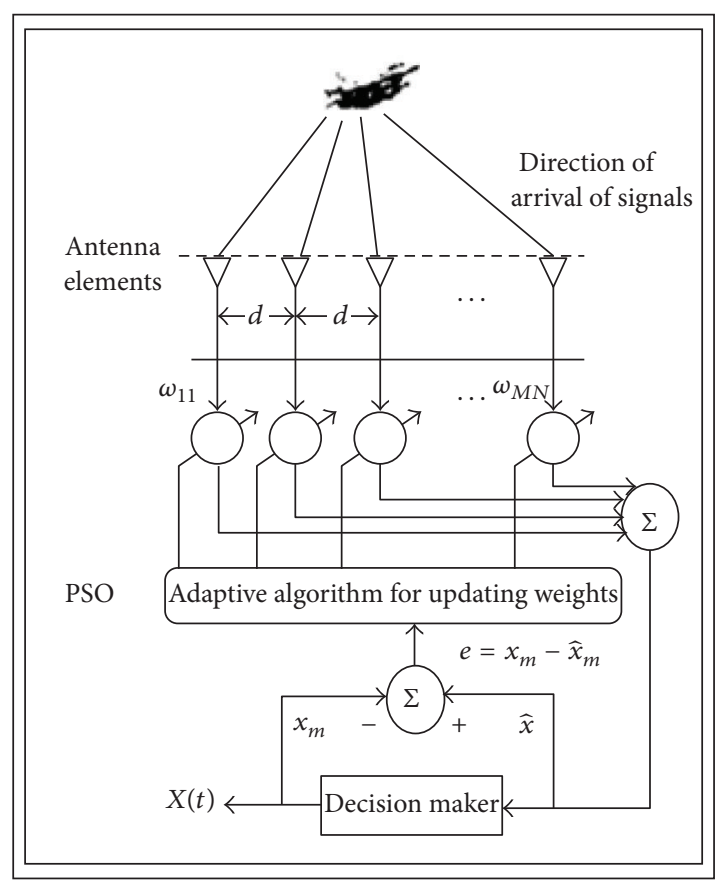

Figure 1: Visualization of system model.

a phase shift. So the phase shift between reference antenna element $\delta_{1}(t)$ and other antenna elements $\delta_{m}(t)$ due to the same source will be expressed as

$$
\Delta_{\delta m}=\delta_{m}(t)-\delta_{1}(t)
$$

General form of (1) in 3D array system is as follows:

$$
\Delta_{\delta m}=-k x_{m} \cos \varphi \sin \varphi-k y_{m} \sin \varphi \sin \theta-k z_{m} \cos \varphi .
$$

But we are using ULA so we only require horizontal plane while $y$ and $z$ coordinates will be truncated. $K=2 \pi / \lambda$, and it is the propagation constant in the free space, while $\lambda$ is the wavelength of the signal, because ULA incoming signals are received horizontally and all elements of ULA are equally distant. Spacing between each element is $\Delta_{x}=d$ and $x_{m}=$ $(m-1) d$. Then $(2)$ can be rewritten along $x$-axis as follows:

$$
\Delta_{\delta m}=-k d(m-1) \cos \varphi
$$

$\Delta \gamma$ is phase shift and zero is taken at the reference antenna element. Now the incoming signal at the antenna element 1 because of $l_{\text {th }}$ source is defined as

$$
\alpha_{1}(t)=m_{1}(t) e^{2 \pi f_{0} t},
$$

where $m_{1}(t)$ is modulating signal of $l_{\text {th }}$ source and $f_{0}$ is carrier frequency. In this case, incoming signal at the $m_{\text {th }}$ element will be

$$
\begin{aligned}
x_{m}(t) & =m_{1}(t) e^{j\left(2 \pi t+\Delta_{\gamma m}\right)}+N_{m}(t) \\
& =\alpha_{1}(t) a_{m}\left(\varphi_{l}\right)+N_{m}(t)
\end{aligned}
$$

where

$$
a_{m}\left(\varphi_{l}\right)=e^{j \Delta_{\gamma m}}=e^{-j k d(m-1) \cos \varphi_{l}} .
$$


$N_{m}(t)$ is denoted the random noise. It consists of external and internal noise produced in the channel. Suppose signal travels in AWGN medium with zero mean and $\sigma_{n}^{2}$ variance. Steering vector of $l_{\text {th }}$ source will be expressed as

$$
a\left(\varphi_{l}\right)=\left[\begin{array}{c}
1 \\
a_{2}\left(\varphi_{l}\right) \\
\vdots \\
a_{m}\left(\varphi_{l}\right) \\
\vdots \\
a_{M}\left(\varphi_{l}\right)
\end{array}\right] .
$$

Now if it is assumed that all sources occur simultaneously then signal at the $m_{\text {th }}$ element will be expressed as

$$
\begin{aligned}
& x_{m}(t)=\sum_{l=1}^{L} m_{1}(t) e^{j\left(2 \pi t+\Delta_{\gamma m}\right)}+N_{m}(t), \\
& x_{m}(t)=\sum_{l=1}^{L} \alpha_{l}(t) a_{m}(\varphi)+N_{m}(t) .
\end{aligned}
$$

$X(t), \alpha(t), N(T)$, and $\varphi_{l}(t)$ are expressed as array signal vector, incoming signal vector, noise vector, and corresponding steering vector, respectively, of $M \times L$ matrix below:

$$
\begin{aligned}
& X(t)=\left\{x_{1}(t), x_{2}(t), x_{3}(t), \ldots, x_{m}(t)\right\}^{T}, \\
& \alpha(t)=\left\{\alpha_{1}(t), \alpha_{2}(t), \alpha_{3}(t), \ldots, \alpha_{m}(t)\right\}^{T}, \\
& N(t)=\left\{n_{1}(t), n_{2}(t), n_{3}(t), \ldots, n_{m}(t)\right\}^{T}, \\
& \varphi(t)=\left\{a_{1}\left(\varphi_{1}\right), a\left(\varphi_{2}\right), a_{3}\left(\varphi_{3}\right), \ldots, a\left(\varphi_{l}\right)\right\}^{T} .
\end{aligned}
$$

Matrix notation of all of these parameters is

$$
X(t)=A \alpha(t)+N(t)
$$

For single snapshot (5) is reduced to the following:

$$
x_{m}(t)=\sum_{k=1}^{m} \alpha_{k} e^{-j k d(m-1) \cos \varphi_{k}}+\theta .
$$

By using this equation, we are able to make fitness function on the basis of mean error square. With the help of this fitness function we estimate amplitude and DOA by applying Particle Swarm Optimization (PSO).

\section{Proposed Methodology}

In this section, overall procedure of the proposed methodology and suggested technique is discussed briefly. All narrow band signals of far field targets are received at antenna elements, processed in RADAR signal processing and optimized

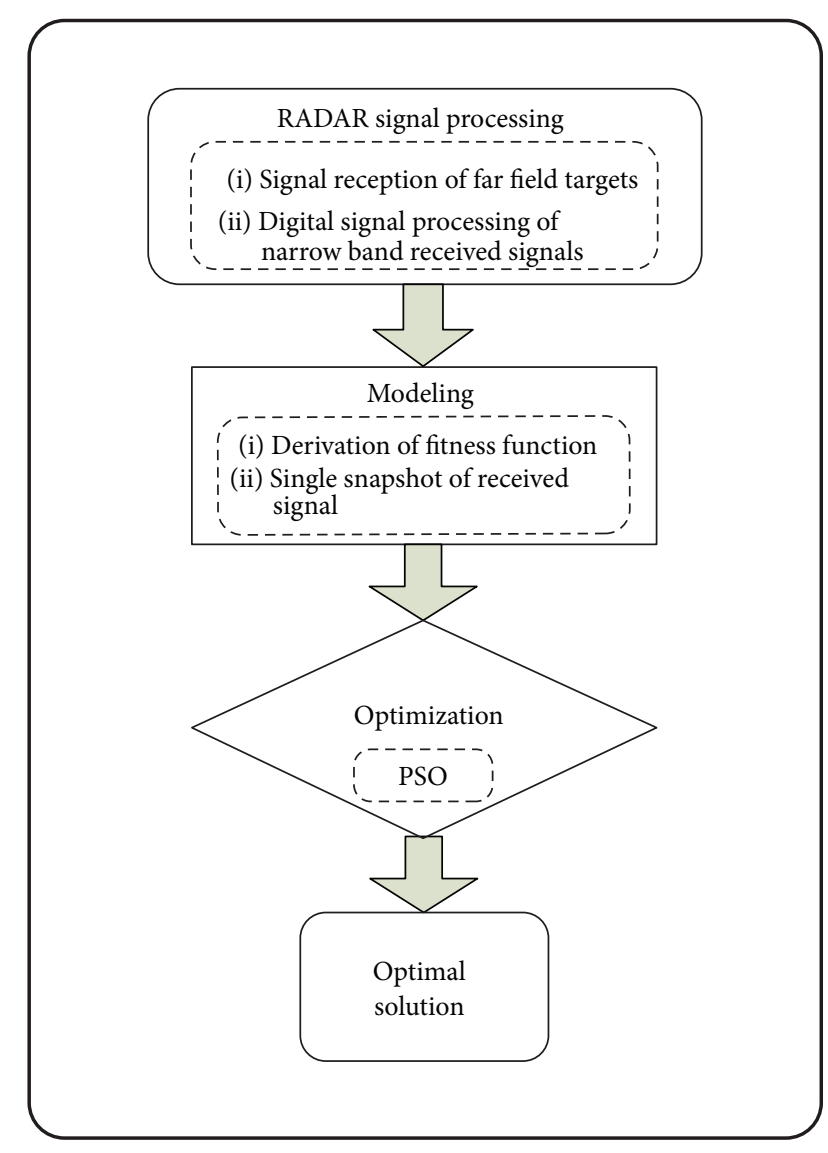

FIGURE 2: Overall performance of proposed methodology.

by Particle Swarm optimization. Flow diagram of the whole process is demonstrated in Figure 2.

4.1. Particle Swarm Optimization. We propose Particle Swarm Optimization for DOA and amplitude estimation. PSO is a heuristic global optimization technique invented by Dr. Kennedy and Eberhart in 1995 [19]. It is population based search algorithm based on the simulation of the social behavior of birds, bees, or fish schooling [20]. Algorithm of PSO is described in two phases as demonstrated in Figure 3: (1) swarm initialization phase (see Section 4.1.1) and (2) swarm execution phase (see Section 4.1.2).

4.1.1. Initialization Phase. Initialization phase consists of three steps:

(a) Step 1: parameter settings:

(i) Determine the particle size (ps) and set the value of other parameters.

(ii) Initialize weight factor.

(iii) Randomly generate the population vector.

(iv) Size of population vector is $\mathrm{pv}=\left[\begin{array}{ll}k_{p} & k_{i}\end{array}\right] m \times 2$.

(v) Substitute the data of $\mathrm{pv}$ in objective function to obtain fitness vector.

(vi) Fitness vector $f v=[] m \times 1$. 


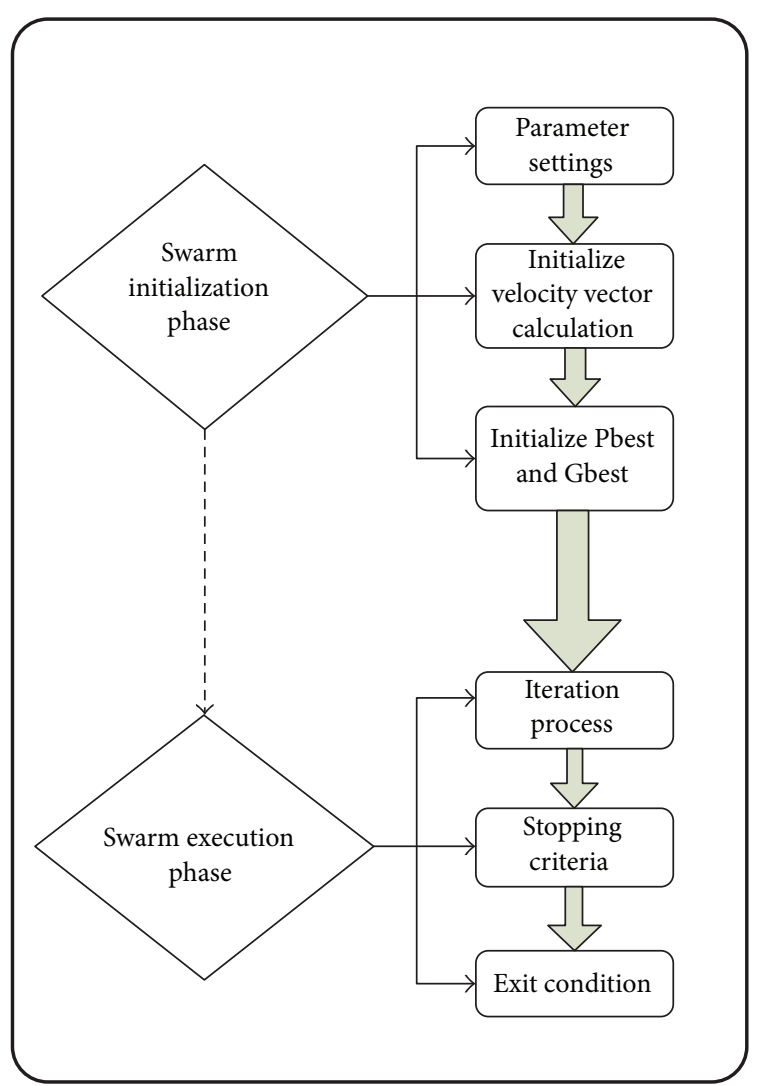

FIGURE 3: Initialization and execution phase of PSO.

(b) Step 2: initialize velocity vector:

(i) In PSO velocity vector is kept in the range $\left[-V_{\max } V_{\max }\right]$.

(ii) $V_{\max }=\left(K_{p} \max -K_{p} \min \right) / n$.

(iii) $\mathrm{vv}=\mathrm{ps} \times \mathrm{ncv}$; ncv stands for number of control variables.

(c) Step 3: initialize Pbest and Gbest:

(i) Pbest $_{\text {population }}=$ Population Vector.

(ii) Pbest $_{\text {fitness }}=$ Fitness vector.

(iii) Gbest is the best position among all individuals.

4.1.2. Execution Phase. Execution phase is also comprised of three steps:

(d) Step 4: iteration process:
(i) weight updating,
(ii) velocity updating,
(iii) position updating.

(e) Step 5: stopping criteria:

(i) Value of fitness function is calculated for each agent in accordance with the above steps.

(ii) Compare fitness of particle evaluation with its Gbest.

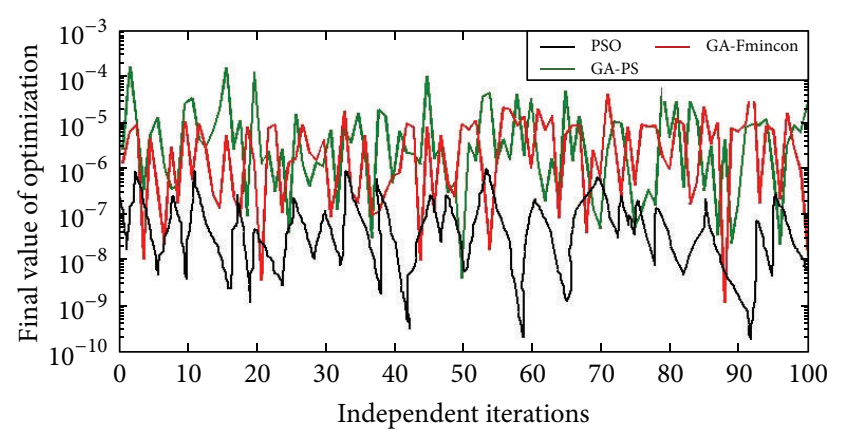

FIGURE 4: Optimized results of PSO compared with GA-PS and GAFmincon for two targets close to each other.

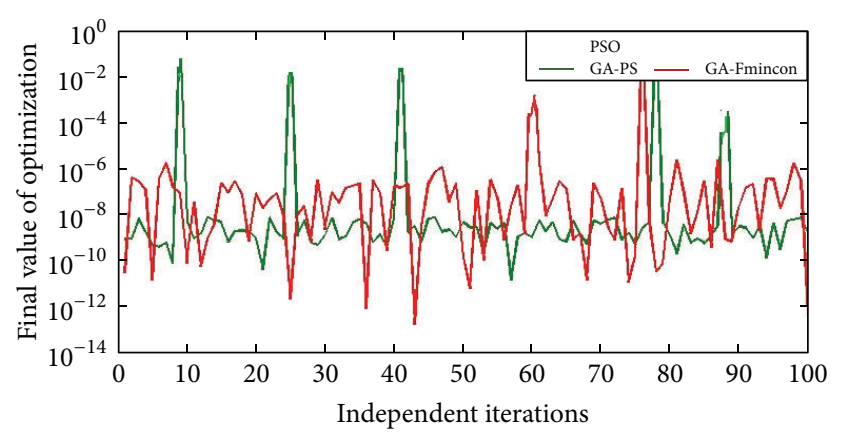

FIGURE 5: Optimized results of PSO compared with GA-PS and GAFmincon for two targets far away from each other.

(f) Step 6: exit condition:

(i) If error $>$ Epbest or a maximum number of iterations run then stop.

(ii) Otherwise go to Step 4 and repeat the process.

\section{Results and Discussion}

In this section, optimized values attained from PSO algorithm along with graphs are described and PSO results have been observed by comparing with GA-PS and GA-Fmincon. Statistical analysis of these schemes is carried out by 100 independent runs and max, mean, and min values are taken from there. These results are categorized into three types and each type contains three scenarios as mentioned in Figure 13.

5.1. PSO Optimized Results Compared with GA-PS and GAFmincon for Type I. When two targets are close to each other PSO converge at $63 \%$ while GA-PS and GA-Fmincon converge at $29 \%$ and $40 \%$ simultaneously at convergence level of $10^{-08}$. Performance of these schemes is compared more clearly in Figure 4 and Table 1.

In this scenario, when two targets are at some distance PSO provides error-free optimized results while GA-PS and GA-Fmincon converge at $87 \%$ and $94 \%$ simultaneously at convergence level of $10^{-08}$. These results are compared more clearly in Figure 5 and Table 2. 
TABLE 1: Optimized results of PSO compared with GA-PS and GA-Fmincon for two targets close to each other.

\begin{tabular}{|c|c|c|c|c|c|c|c|c|c|}
\hline & \multirow{2}{*}{ Schemes } & \multirow{2}{*}{ Mode } & \multicolumn{2}{|c|}{ Amplitudes } & \multicolumn{2}{|c|}{ Phases } & \multirow{2}{*}{ Error } & \multirow{2}{*}{ MSE } & \multirow{2}{*}{$\begin{array}{c}\text { Convergence } \\
\varepsilon \geq 10^{-08}\end{array}$} \\
\hline & & & $\propto_{1}$ & $\propto_{2}$ & $\varphi_{1}$ & $\varphi_{2}$ & & & \\
\hline \multirow{10}{*}{ Scenario I } & \multirow{3}{*}{ PSO } & Max & 0.9964 & 2.0095 & 0.5161 & 0.5731 & $4.97 E-10$ & \multirow{3}{*}{$2.03 E-07$} & \multirow{3}{*}{$63 \%$} \\
\hline & & Mean & 1.0159 & 1.9841 & 0.5314 & 0.5822 & $5.79 E-08$ & & \\
\hline & & Min & 0.8660 & 2.2339 & 0.5447 & 0.5893 & $1.00 E-06$ & & \\
\hline & \multirow{3}{*}{ GA-PS } & Max & 1.0002 & 2.0008 & 0.5238 & 0.5760 & $3.97 E-09$ & \multirow{3}{*}{$1.19 E-05$} & \multirow{3}{*}{$29 \%$} \\
\hline & & Mean & 1.0012 & 2.0024 & 0.5225 & 0.5764 & $6.10 E-06$ & & \\
\hline & & Min & 1.1104 & 2.0858 & 0.6752 & 0.5555 & $1.42 E-04$ & & \\
\hline & \multirow{3}{*}{ GA-Fmincon } & Max & 1.0070 & 2.0000 & 0.5238 & 0.5760 & $6.00 E-09$ & \multirow{3}{*}{$5.77 E-06$} & \multirow{3}{*}{$40 \%$} \\
\hline & & Mean & 1.0018 & 2.0081 & 0.5149 & 0.5727 & $9.06 E-07$ & & \\
\hline & & Min & 1.0565 & 2.0419 & 0.6583 & 0.5542 & $9.65 E-05$ & & \\
\hline & True value & & 1.0000 & 2.0000 & 0.5236 & 0.5760 & & & \\
\hline
\end{tabular}

TABLE 2: Optimized results of PSO compared with GA-PS and GA-Fmincon for two targets far away from each other.

\begin{tabular}{|c|c|c|c|c|c|c|c|c|c|}
\hline & \multirow{2}{*}{ Schemes } & \multirow{2}{*}{ Mode } & \multicolumn{2}{|c|}{ Amplitudes } & \multicolumn{2}{|c|}{ Phases } & \multirow{2}{*}{ Error } & \multirow{2}{*}{ MSE } & \multirow{2}{*}{$\begin{array}{c}\text { Convergence } \\
\varepsilon \geq 10^{-08}\end{array}$} \\
\hline & & & $\propto_{1}$ & $\propto_{2}$ & $\varphi_{1}$ & $\varphi_{2}$ & & & \\
\hline \multirow{10}{*}{ Scenario II } & \multirow{3}{*}{ PSO } & Max & 1.0000 & 2.0000 & 1.0472 & 2.0944 & No Error & \multirow{3}{*}{ - } & \multirow{3}{*}{$100 \%$} \\
\hline & & Mean & 1.0000 & 2.0000 & 1.0472 & 2.0944 & No Error & & \\
\hline & & Min & 1.0000 & 2.0000 & 1.0472 & 2.0944 & No Error & & \\
\hline & \multirow{3}{*}{ GA-PS } & Max & 1.0000 & 2.0009 & 1.0471 & 2.0943 & $8.39 E-11$ & \multirow{3}{*}{$4.78 E-02$} & \multirow{3}{*}{$87 \%$} \\
\hline & & Mean & 1.0001 & 2.0017 & 1.0471 & 2.0943 & $5.49 E-06$ & & \\
\hline & & Min & 1.0999 & 2.0035 & 1.3471 & 2.7943 & $1.03 E-01$ & & \\
\hline & \multirow{3}{*}{ GA-Fmincon } & Max & 1.0000 & 2.0009 & 1.0471 & 2.0943 & $2.37 E-12$ & \multirow{3}{*}{$1.91 E-02$} & \multirow{3}{*}{$94 \%$} \\
\hline & & Mean & 1.0000 & 2.0016 & 1.0472 & 2.0943 & $5.52 E-07$ & & \\
\hline & & Min & 1.0996 & 2.0001 & 1.2472 & 2.3944 & $1.33 E-02$ & & \\
\hline & True value & & 1.0000 & 2.0000 & 1.0472 & 2.0944 & & & \\
\hline
\end{tabular}

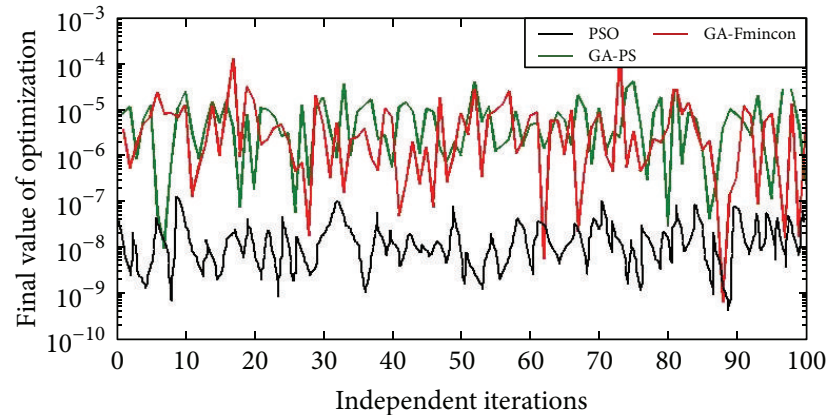

FIGURE 6: Optimized results of PSO compared with GA-PS and GAFmincon for two targets close to the surface of earth.

When two targets are moving at low altitude then PSO converges at $91 \%$ at convergence level of $10^{-08}$, while GA-PS and GA-Fmincon converge at $74 \%$ and $85 \%$ simultaneously in this scenario (Table 3). Assessment diagram of these three optimization schemes is depicted in Figure 6.

5.2. PSO Optimized Results Compared with GA-PS and GAFmincon for Type II. In Table 4 of type II three targets

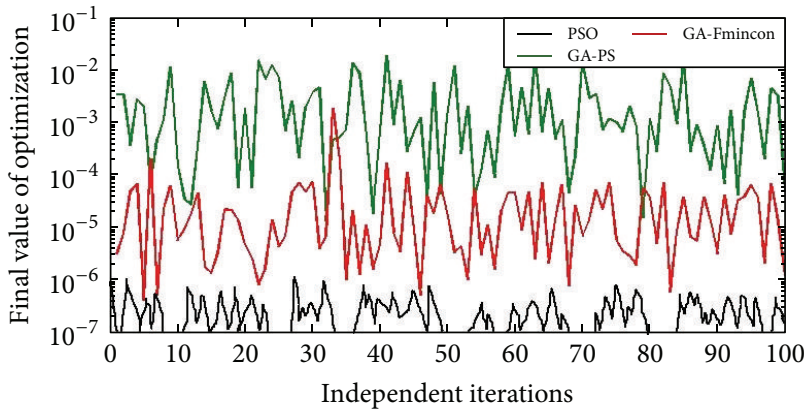

FIGURE 7: Optimized results of PSO compared with GA-PS and GAFmincon for three targets close to each other.

are optimized at very short distance to each other. PSO, GA-PS, and GA-Fmincon converge at $47 \%, 19 \%$, and $30 \%$ concurrently at convergence level of $10^{-07}$. Figure 7 describes the overall performance of these three schemes.

When three targets are at some distance to each other PSO converge at $100 \%$ at $10^{-07}$ convergence level. GA-PS and GAFmincon converge at $36 \%$ and $45 \%$ at the same time. Figure 8 and Table 5 show the combined performance of these three schemes. 
TABLE 3: Optimized results of PSO compared with GA-PS and GA-Fmincon for two targets close to the surface of earth.

\begin{tabular}{|c|c|c|c|c|c|c|c|c|c|}
\hline & \multirow{2}{*}{ Schemes } & \multirow{2}{*}{ Mode } & \multicolumn{2}{|c|}{ Amplitudes } & \multicolumn{2}{|c|}{ Phases } & \multirow{2}{*}{ Error } & \multirow{2}{*}{ MSE } & \multirow{2}{*}{$\begin{array}{c}\text { Convergence } \\
\varepsilon \geq 10^{-08}\end{array}$} \\
\hline & & & $\propto_{1}$ & $\propto_{2}$ & $\varphi_{1}$ & $\varphi_{2}$ & & & \\
\hline \multirow{9}{*}{ Scenario III } & \multirow{3}{*}{ PSO } & Max & 1.0007 & 1.9793 & 0.1294 & 2.5791 & $3.81 E-09$ & \multirow{3}{*}{$5.37 E-08$} & \multirow{3}{*}{$91 \%$} \\
\hline & & Mean & 1.0159 & 1.8841 & 0.3922 & 2.5414 & $5.79 E-08$ & & \\
\hline & & Min & 0.8961 & 2.1339 & 0.4993 & 2.5447 & $1.00 E-07$ & & \\
\hline & \multirow{3}{*}{ GA-PS } & $\operatorname{Max}$ & 1.0001 & 2.0009 & 0.1744 & 2.6159 & $9.04 E-08$ & \multirow{3}{*}{$8.30 E-06$} & \multirow{3}{*}{$74 \%$} \\
\hline & & Mean & 1.0035 & 2.0060 & 0.1190 & 2.5739 & $5.42 E-07$ & & \\
\hline & & Min & 1.1345 & 2.0635 & 0.4298 & 0.5636 & $6.47 E-04$ & & \\
\hline & \multirow{3}{*}{ GA-Fmincon } & Max & 1.0001 & 2.0006 & 0.1745 & 2.6159 & $6.05 E-08$ & \multirow{3}{*}{$7.75 E-06$} & \multirow{3}{*}{$85 \%$} \\
\hline & & Mean & 1.0029 & 2.0066 & 0.1762 & 2.5731 & $7.55 E-07$ & & \\
\hline & & Min & 0.9951 & 2.0146 & 0.4516 & 2.5647 & $3.92 E-04$ & & \\
\hline & True value & & 1.0000 & 2.00000 & 0.1745 & 2.9671 & & & \\
\hline
\end{tabular}

TABLE 4: Optimized results of PSO compared with GA-PS and GA-Fmincon for three targets close to each other.

\begin{tabular}{|c|c|c|c|c|c|c|c|c|c|c|c|}
\hline & \multirow{2}{*}{ Schemes } & \multirow{2}{*}{ Mode } & \multicolumn{3}{|c|}{ Amplitudes } & \multicolumn{3}{|c|}{ Phases } & \multirow{2}{*}{ Error } & \multirow{2}{*}{ MSE } & \multirow{2}{*}{$\begin{array}{c}\text { Convergence } \\
\varepsilon \geq 10^{-07}\end{array}$} \\
\hline & & & $\propto_{1}$ & $\propto_{2}$ & $\propto_{3}$ & $\varphi_{1}$ & $\varphi_{2}$ & $\varphi_{3}$ & & & \\
\hline \multirow{9}{*}{ Scenario I } & \multirow{3}{*}{ PSO } & $\operatorname{Max}$ & 0.3087 & 1.9286 & 3.0627 & 0.5432 & 0.5422 & 0.6234 & $5.22 E-09$ & & \\
\hline & & Mean & 1.0506 & 2.1322 & 3.0638 & 0.5530 & 0.5534 & 0.6302 & $5.86 E-07$ & $7.02 E-06$ & $47 \%$ \\
\hline & & Min & 1.0826 & 2.1120 & 3.3621 & 0.4949 & 0.6260 & 0.5989 & $3.16 E-05$ & & \\
\hline & \multirow{3}{*}{ GA-PS } & $\operatorname{Max}$ & 1.0000 & 2.0001 & 3.0009 & 1.5160 & 0.5774 & 0.6258 & $1.49 E-05$ & & \\
\hline & & Mean & 1.0027 & 2.0414 & 3.0078 & 1.5111 & 1.6138 & 1.6001 & $8.36 E-03$ & $2.92 E-03$ & $19 \%$ \\
\hline & & Min & 1.0669 & 1.9398 & 2.9998 & 1.5508 & 1.6429 & 1.5824 & $1.41 E-02$ & & \\
\hline & \multirow{3}{*}{ GA-Fmincon } & $\operatorname{Max}$ & 1.0004 & 2.0002 & 3.0004 & 0.5157 & 0.5790 & 0.6215 & $3.90 E-07$ & & \\
\hline & & Mean & 1.0008 & 2.0069 & 3.0684 & 1.5150 & 1.5346 & 1.6048 & $4.45 E-05$ & $4.42 E-05$ & $30 \%$ \\
\hline & & Min & 1.0475 & 2.0502 & 3.0891 & 1.6227 & 1.6224 & 1.5461 & $1.78 E-03$ & & \\
\hline & True value & & 1.0000 & 2.0000 & 3.0000 & 0.5236 & 0.5760 & 0.6283 & & & \\
\hline
\end{tabular}

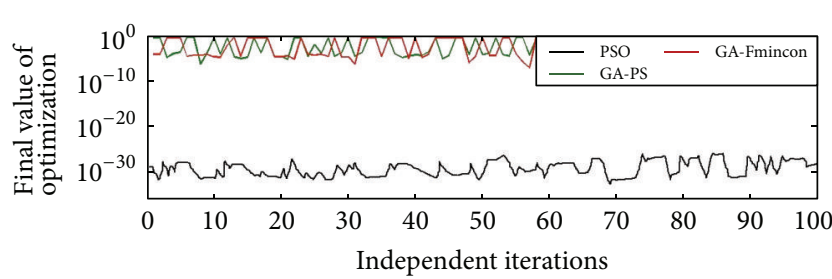

FIGURE 8: Optimized results of PSO compared with GA-PS and GAFmincon for three targets far away from each other.

When three targets are near the surface of earth PSO converges at $61 \%$, GA-PS converges at $20 \%$, and GA-Fmincon converges at $54 \%$ at convergence level of $10^{-07}$. In this situation PSO again perform well (Table 6). In Figure 9 all of these results are shown graphically.

5.3. PSO Optimized Results Compared with GA-PS and GAFmincon for Type III. In Scenario 1 of type III four targets are optimized with very short distance to each other. PSO converges at $63 \%$, GA-PS converges at $12 \%$, and GA-Fmincon converges at $45 \%$ at convergence level of $10^{-07}$ in this scenario (Table 7). Graphical representation of these schemes is depicted in Figure 10.

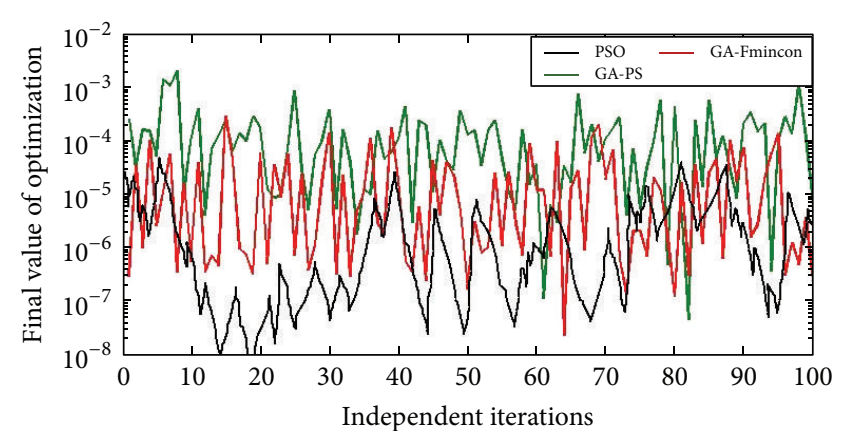

FIgURE 9: Optimized results of PSO compared with GA-PS and GAFmincon for three targets close to the surface of earth.

When four targets are far away from each other then PSO provides $83 \%$ convergence, GA-PS converges at $65 \%$, and GAFmincon converges at $71 \%$ at the convergence level of $10^{-07}$. Figure 11 shows graphs of these results given in Table 8 .

In Table 9 optimization results of four targets at very low altitude are shown. In this scenario, PSO converges at 70\%, GA-PS converges at $61 \%$, and GA-Fmincon converges at $51 \%$ at the convergence level of $10^{-07}$. Graphically these results are shown in Figure 12. 
TABLE 5: Optimized results of PSO compared with GA-PS and GA-Fmincon for three targets far away from each other.

\begin{tabular}{|c|c|c|c|c|c|c|c|c|c|c|c|}
\hline & \multirow{2}{*}{ Schemes } & \multirow{2}{*}{ Mode } & \multicolumn{3}{|c|}{ Amplitudes } & \multicolumn{3}{|c|}{ Phases } & \multirow{2}{*}{ Error } & \multirow{2}{*}{ MSE } & \multirow{2}{*}{$\begin{array}{c}\text { Convergence } \\
\varepsilon \geq 10^{-07}\end{array}$} \\
\hline & & & $\propto_{1}$ & $\propto_{2}$ & $\propto_{3}$ & $\varphi_{1}$ & $\varphi_{2}$ & $\varphi_{3}$ & & & \\
\hline \multirow{9}{*}{ Scenario II } & \multirow{3}{*}{ PSO } & $\operatorname{Max}$ & 1.0000 & 2.0000 & 3.0000 & 1.0471 & 1.5708 & 2.0943 & $2.47 E-32$ & \multirow{3}{*}{$2.07 E-30$} & \multirow{3}{*}{$100 \%$} \\
\hline & & Mean & 1.0000 & 2.0000 & 3.0000 & 1.0472 & 1.5706 & 2.0888 & $5.50 E-31$ & & \\
\hline & & Min & 1.0000 & 2.0000 & 3.0000 & 1.0471 & 1.5707 & 2.1887 & $5.50 E-30$ & & \\
\hline & \multirow{3}{*}{ GA-PS } & $\operatorname{Max}$ & 1.0004 & 2.0009 & 3.0005 & 0.7850 & 1.5708 & 2.0947 & $2.12 E-07$ & \multirow{3}{*}{$7.60 E-02$} & \multirow{3}{*}{$36 \%$} \\
\hline & & Mean & 1.0067 & 2.0094 & 3.0081 & 0.7885 & 1.5711 & 2.0902 & $1.11 E-04$ & & \\
\hline & & Min & 1.0369 & 2.0172 & 3.0835 & 0.7000 & 1.5737 & 2.0000 & $1.55 E-01$ & & \\
\hline & \multirow{3}{*}{ GA-Fmincon } & Max & 1.0007 & 2.0000 & 3.0002 & 0.7851 & 1.5707 & 2.0949 & $3.34 E-08$ & \multirow{3}{*}{$8.04 E-02$} & \multirow{3}{*}{$45 \%$} \\
\hline & & Mean & 1.0059 & 2.0097 & 3.0002 & 0.7802 & 1.5708 & 2.0910 & $1.36 E-05$ & & \\
\hline & & Min & 1.0492 & 2.1679 & 3.0204 & 0.7737 & 1.5737 & 2.0000 & $1.55 E-01$ & & \\
\hline & True value & & 1.0000 & 2.0000 & 3.0000 & 0.5236 & 1.0472 & 2.0944 & & & \\
\hline
\end{tabular}

TABLE 6: Optimized results of PSO compared with GA-PS and GA-Fmincon for three targets close to the surface of earth.

\begin{tabular}{|c|c|c|c|c|c|c|c|c|c|c|c|}
\hline & \multirow{2}{*}{ Schemes } & \multirow{2}{*}{ Mode } & \multicolumn{3}{|c|}{ Amplitudes } & \multicolumn{3}{|c|}{ Phases } & \multirow{2}{*}{ Error } & \multirow{2}{*}{ MSE } & \multirow{2}{*}{$\begin{array}{c}\text { Convergence } \\
\varepsilon \geq 10^{-07}\end{array}$} \\
\hline & & & $\propto_{1}$ & $\propto_{2}$ & $\propto_{3}$ & $\varphi_{1}$ & $\varphi_{2}$ & $\varphi_{3}$ & & & \\
\hline \multirow{9}{*}{ Scenario III } & \multirow{3}{*}{ PSO } & $\operatorname{Max}$ & 1.0413 & 2.0000 & 3.0035 & 1.5772 & 1.5772 & 2.6148 & $2.50 E-09$ & \multirow{3}{*}{$3.01 E-08$} & \multirow{3}{*}{$61 \%$} \\
\hline & & Mean & 1.1712 & 1.8956 & 2.8288 & 1.3792 & 1.5708 & 2.0405 & $1.37 E-07$ & & \\
\hline & & Min & 1.2120 & 2.2337 & 2.3102 & 0.1938 & 1.1640 & 2.5708 & $4.32 E-04$ & & \\
\hline & \multirow{3}{*}{ GA-PS } & $\operatorname{Max}$ & 1.0008 & 1.9999 & 3.0008 & 0.1720 & 1.5725 & 2.9671 & $1.13 E-07$ & \multirow{3}{*}{$1.76 E-04$} & \multirow{3}{*}{$20 \%$} \\
\hline & & Mean & 1.0016 & 1.9965 & 3.9786 & 0.1582 & 1.5273 & 2.8861 & $5.73 E-05$ & & \\
\hline & & Min & 1.2909 & 2.2747 & 3.4214 & 0.2619 & 1.5964 & 2.5735 & $2.00 E-03$ & & \\
\hline & \multirow{3}{*}{ GA-Fmincon } & Max & 1.0008 & 2.0000 & 3.0000 & 0.1749 & 1.5724 & 2.9676 & $2.31 E-08$ & \multirow{3}{*}{$2.69 E-05$} & \multirow{3}{*}{$54 \%$} \\
\hline & & Mean & 1.0073 & 1.9999 & 2.9999 & 0.1368 & 1.5347 & 2.8644 & $3.77 E-06$ & & \\
\hline & & Min & 0.9388 & 1.8999 & 3.0206 & 0.2424 & 1.5399 & 2.5598 & $1.35 E-04$ & & \\
\hline & True value & & 1.0000 & 2.0000 & 3.0000 & 0.1745 & 1.5708 & 2.9671 & & & \\
\hline
\end{tabular}

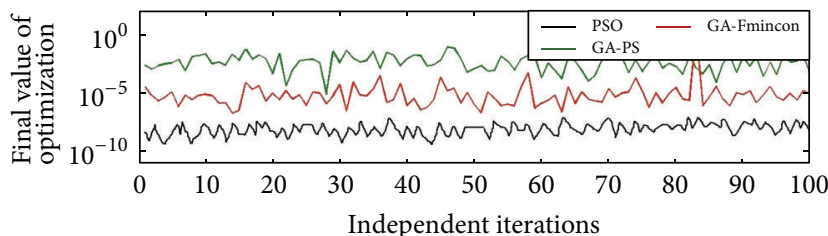

FIGURE 10: Optimized results of PSO compared with GA-PS and GA-Fmincon for four targets close to each other.

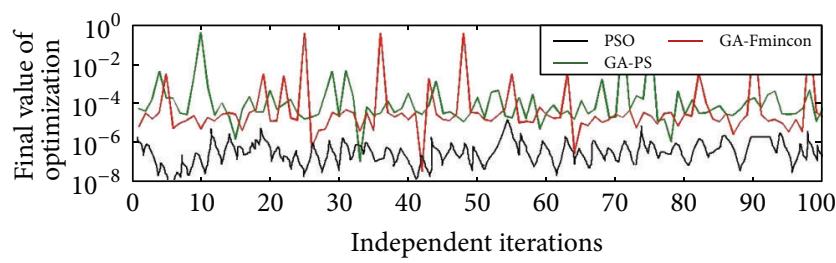

FIGURE 11: Optimized results of PSO compared with GA-PS and GAFmincon for four targets far away from each other.

\section{Comparison with Existing Techniques}

In Table 10 PSO is generally compared with existing hybridized algorithms in accordance with estimation of DOA and amplitude. Effectiveness of the algorithm is illustrated

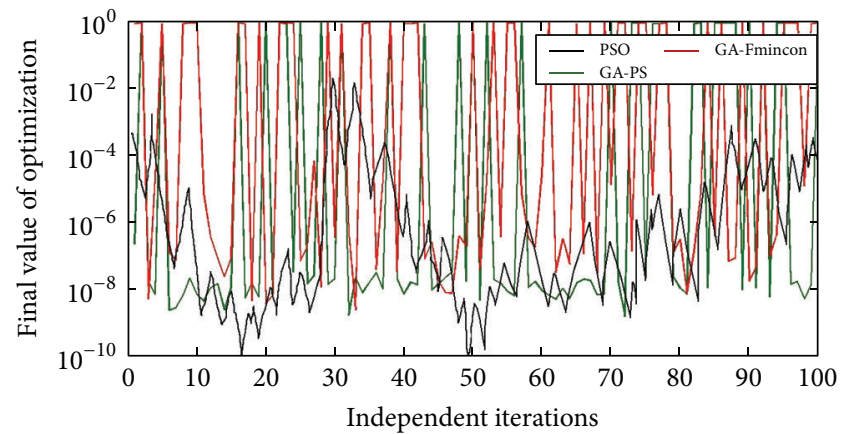

FIGURE 12: Optimized results of PSO compared with GA-PS and GAFmincon for four targets close to the surface of earth.

in Table 10. Performance of the proposed algorithm is much better than other existing techniques. Average MSE, convergence rate, and error depicted in this table are average value of the results in Section 5.

\section{Conclusions and Future Recommendations}

On the basis of results and discussions portion we made up the following conclusions. 
TABLE 7: Optimized results of PSO compared with GA-PS and GA-Fmincon for four targets close to each other.

\begin{tabular}{|c|c|c|c|c|c|c|c|c|c|c|c|c|c|}
\hline & \multirow{2}{*}{ Schemes } & \multirow{2}{*}{ Mode } & \multicolumn{4}{|c|}{ Amplitudes } & \multicolumn{4}{|c|}{ Phases } & \multirow{2}{*}{ Error } & \multirow{2}{*}{ MSE } & \multirow{2}{*}{$\begin{array}{c}\text { Convergence } \\
\varepsilon \geq 10^{-07}\end{array}$} \\
\hline & & & $\propto_{1}$ & $\propto_{2}$ & $\propto_{3}$ & $\propto_{4}$ & $\varphi_{1}$ & $\varphi_{2}$ & $\varphi_{3}$ & $\varphi_{4}$ & & & \\
\hline \multirow{9}{*}{ Scenario I } & \multirow{3}{*}{ PSO } & $\operatorname{Max}$ & 0.9527 & 2.0834 & 3.1068 & 3.7570 & 0.5419 & 0.7066 & 0.6293 & 0.6691 & $7.47 E-09$ & \multirow{3}{*}{$5.07 E-07$} & \multirow{3}{*}{$63 \%$} \\
\hline & & Mean & 1.5734 & 2.4055 & 3.2595 & 4.2525 & 0.5284 & 0.6195 & 0.6174 & 0.6864 & $2.52 E-08$ & & \\
\hline & & Min & 0.7770 & 1.5646 & 3.5882 & 4.6092 & 0.4723 & 0.5222 & 0.6494 & 0.6917 & $1.13 E-06$ & & \\
\hline & \multirow{3}{*}{ GA-PS } & Max & 0.3279 & 2.1876 & 3.7090 & 3.7734 & 0.2699 & 0.1125 & 0.2157 & 0.2064 & $7.00 E-07$ & \multirow{3}{*}{$9.10 E-04$} & \multirow{3}{*}{$12 \%$} \\
\hline & & Mean & 1.4329 & 2.0503 & 2.8081 & 3.7207 & 1.0952 & 1.1734 & 1.2697 & 1.2729 & $4.27 E-04$ & & \\
\hline & & Min & 0.4281 & 1.5860 & 3.6188 & 4.5455 & 1.0089 & 2.1716 & 1.2924 & 2.1828 & $4.31 E-02$ & & \\
\hline & \multirow{3}{*}{ GA-Fmincon } & $\operatorname{Max}$ & 1.5488 & 2.0299 & 2.9026 & 3.5184 & 0.1025 & 0.2752 & 0.2085 & 0.2504 & $1.90 E-07$ & \multirow{3}{*}{$1.89 E-03$} & \multirow{3}{*}{$45 \%$} \\
\hline & & Mean & 1.0795 & 2.5464 & 2.7853 & 3.5750 & 1.2735 & 0.1194 & 0.1774 & 1.2605 & $2.12 E-04$ & & \\
\hline & & Min & 0.3450 & 1.8918 & 3.1111 & 4.6440 & 1.1146 & 1.1143 & 2.2010 & 2.2651 & $1.89 E+0$ & & \\
\hline \multicolumn{3}{|c|}{ True value } & 1 & 2 & 3 & 4 & 0.5236 & 0.5760 & 0.6283 & 0.6807 & & & \\
\hline
\end{tabular}

TABLE 8: Optimized results of PSO compared with GA-PS and GA-Fmincon for four targets far away from each other.

\begin{tabular}{|c|c|c|c|c|c|c|c|c|c|c|c|c|c|}
\hline & \multirow{2}{*}{ Schemes } & \multirow{2}{*}{ Mode } & \multicolumn{4}{|c|}{ Amplitudes } & \multicolumn{4}{|c|}{ Phases } & \multirow{2}{*}{ Error } & \multirow{2}{*}{ MSE } & \multirow{2}{*}{$\begin{array}{c}\text { Convergence } \\
\varepsilon \geq 10^{-07}\end{array}$} \\
\hline & & & $\propto_{1}$ & $\propto_{2}$ & $\propto_{3}$ & $\propto_{4}$ & $\varphi_{1}$ & $\varphi_{2}$ & $\varphi_{3}$ & $\varphi_{4}$ & & & \\
\hline \multirow{9}{*}{ Scenario II } & \multirow{3}{*}{ PSO } & Max & 1.0546 & 2.1604 & 2.9764 & 4.0004 & 0.6107 & 1.0768 & 1.7402 & 2.0000 & $6.65 E-09$ & \multirow{3}{*}{$2.04 E-07$} & \multirow{3}{*}{$83 \%$} \\
\hline & & Mean & 1.6566 & 2.2770 & 3.1192 & 4.0002 & 0.4766 & 1.4695 & 1.7458 & 2.5198 & $8.89 E-07$ & & \\
\hline & & Min & 1.6566 & 2.6770 & 3.4119 & 3.5499 & 0.3766 & 1.7695 & 1.9182 & 2.5363 & $8.89 E-05$ & & \\
\hline & \multirow{3}{*}{ GA-PS } & $\operatorname{Max}$ & 1.0006 & 2.0001 & 3.0002 & 4.1193 & 0.5234 & 1.0472 & 1.7458 & 2.5313 & $9.49 E-07$ & \multirow{3}{*}{$1.03 E-03$} & \multirow{3}{*}{$65 \%$} \\
\hline & & Mean & 1.0094 & 2.0069 & 3.0021 & 4.5499 & 0.5244 & 1.0463 & 1.7499 & 2.5821 & $1.40 E-03$ & & \\
\hline & & Min & 1.0842 & 1.9006 & 3.0841 & 4.8940 & 1.9258 & 1.9132 & 1.0507 & 2.0000 & $3.40 E-01$ & & \\
\hline & \multirow{3}{*}{ GA-Fmincon } & $\operatorname{Max}$ & 1.0006 & 2.0000 & 3.0000 & 4.0003 & 0.5237 & 1.0471 & 2.4702 & 2.8361 & $2.95 E-08$ & \multirow{3}{*}{$1.69 E-04$} & \multirow{3}{*}{$71 \%$} \\
\hline & & Mean & 1.0007 & 2.0094 & 3.0400 & 4.0008 & 0.5272 & 0.0899 & 2.3195 & 2.9023 & $1.20 E-05$ & & \\
\hline & & Min & 1.0984 & 2.0834 & 3.0770 & 4.1944 & 0.0000 & 1.0506 & 2.3195 & 3.0023 & $3.37 E-01$ & & \\
\hline \multicolumn{3}{|c|}{ True value } & 1 & 2 & 3 & 4 & 0.5236 & 1.0472 & 1.5708 & 2.0944 & & & \\
\hline
\end{tabular}

TABLE 9: Optimized results of PSO compared with GA-Fmincon and GA-PS for four targets close to the surface of earth.

\begin{tabular}{|c|c|c|c|c|c|c|c|c|c|c|c|c|c|}
\hline & \multirow{2}{*}{ Schemes } & \multirow{2}{*}{ Mode } & \multicolumn{4}{|c|}{ Amplitudes } & \multicolumn{4}{|c|}{ Phases } & \multirow{2}{*}{ Error } & \multirow{2}{*}{ MSE } & \multirow{2}{*}{$\begin{array}{c}\text { Convergence } \\
\varepsilon \geq 10^{-07}\end{array}$} \\
\hline & & & $\propto_{1}$ & $\propto_{2}$ & $\propto_{3}$ & $\propto_{4}$ & $\varphi_{1}$ & $\varphi_{2}$ & $\varphi_{3}$ & $\varphi_{4}$ & & & \\
\hline \multirow{9}{*}{ Scenario III } & \multirow{3}{*}{ PSO } & $\operatorname{Max}$ & 1.2025 & 2.0008 & 3.0002 & 3.5963 & 0.7077 & 1.0444 & 2.0471 & 2.9207 & $5.80 E-09$ & \multirow{3}{*}{$1.71 E-06$} & \multirow{3}{*}{$70 \%$} \\
\hline & & Mean & 0.8158 & 2.0306 & 3.0197 & 4.5563 & 0.0808 & 1.0422 & 2.0401 & 2.6860 & $1.19 E-05$ & & \\
\hline & & Min & 1.9351 & 2.0787 & 3.0524 & 4.9367 & 1.1900 & 1.0581 & 2.0000 & 2.1045 & $4.07 E-02$ & & \\
\hline & \multirow{3}{*}{ GA-PS } & Max & 1.0000 & 2.0000 & 3.0000 & 4.0000 & 0.1736 & 1.0490 & 2.0199 & 2.9680 & $2.37 E-08$ & \multirow{3}{*}{$2.31 E-05$} & \multirow{3}{*}{$61 \%$} \\
\hline & & Mean & 1.0008 & 1.9986 & 2.9975 & 3.9996 & 0.5234 & 1.3091 & 1.9198 & 2.6180 & $1.35 E-05$ & & \\
\hline & & Min & 1.3686 & 2.1320 & 2.6706 & 3.0771 & 2.6068 & 1.3112 & 1.6070 & 1.9163 & $8.81 E-01$ & & \\
\hline & \multirow{3}{*}{ GA-Fmincon } & Max & 1.0000 & 2.0000 & 3.0000 & 4.0000 & 0.1736 & 1.0490 & 2.1199 & 2.9680 & $3.60 E-08$ & \multirow{3}{*}{$3.61 E-04$} & \multirow{3}{*}{$51 \%$} \\
\hline & & Mean & 1.0025 & 1.9987 & 3.0023 & 4.0021 & 0.5233 & 1.3090 & 1.9199 & 2.6180 & $1.67 E-05$ & & \\
\hline & & Min & 0.2903 & 2.1427 & 3.0767 & 4.0281 & 3.1408 & 1.3121 & 1.9149 & 2.6017 & $8.17 E-01$ & & \\
\hline \multicolumn{3}{|c|}{ True value } & 1 & 2 & 3 & 4 & 0.1745 & 1.0472 & 2.0944 & 2.9671 & & & \\
\hline
\end{tabular}

TABLE 10: Comparison of PSO and other popular existing techniques.

\begin{tabular}{|c|c|c|c|c|}
\hline \multirow{2}{*}{ S number } & \multirow{2}{*}{ Parameters of comparison } & \multirow{2}{*}{$\begin{array}{c}\text { Proposed methodology } \\
\text { PSO }\end{array}$} & \multicolumn{2}{|c|}{ Other existing techniques } \\
\hline & & & GA-Fmincon & GA-PS \\
\hline 1 & Average MSE & $1.7 E-09$ & $2.3 E-05$ & $3.7 E-04$ \\
\hline 2 & Average convergence rate & $75.33 \%$ & $51.55 \%$ & $44.77 \%$ \\
\hline 3 & Average error & $2.07 E-10$ & $3.71 E-6$ & $5.03 E-5$ \\
\hline 4 & Hardware implementation & Easy & Hard & Hard \\
\hline 5 & System complexity & Simple & Complex & Complex \\
\hline
\end{tabular}




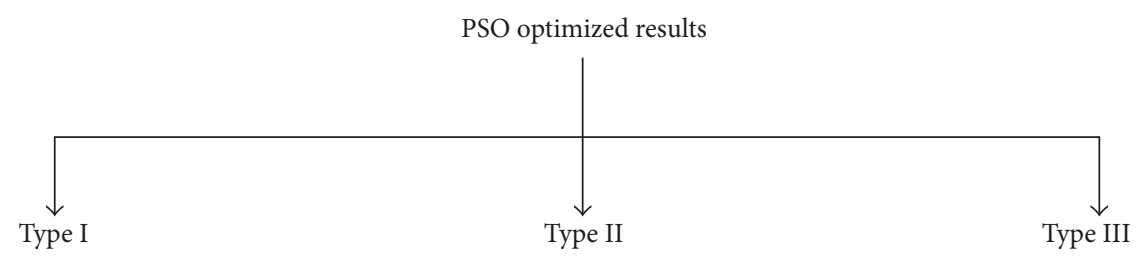

(Optimization of two targets in the far field of antenna array)

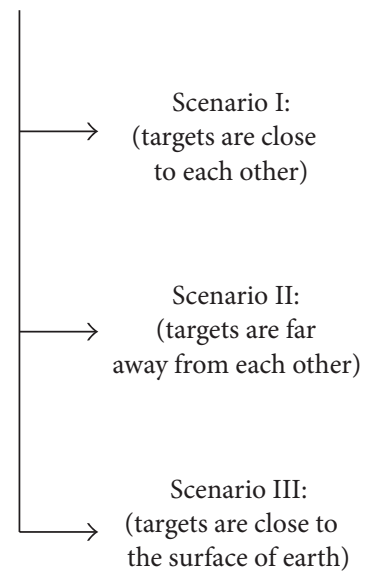

(Optimization of three targets in the far field of antenna array)

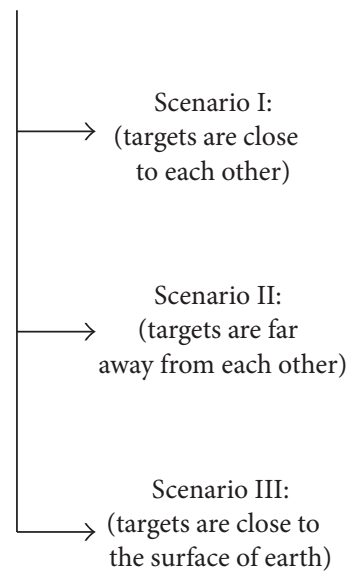

(Optimization of four targets in the far field of antenna array)

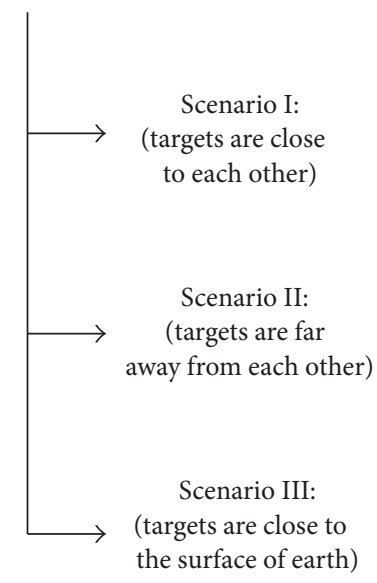

FIGURE 13

We estimate amplitude and DOA of targets in three types of scenarios using PSO technique and compare it with two other GA hybrid schemes, GA-PS and GA-Fmincon. In all three scenarios performance of PSO is better than GA-PS and GA-Fmincon. Besides that, PSO provide accurate and convergent results, the other inherent factor of this scheme is simplicity in concept and being easy to implement in hardware.

In the future one can apply other biological inspired methods like Ant Colony Optimization or Active Set Algorithm for this problem using circular or rectangular shape of antenna arrays.

\section{Conflict of Interests}

The authors declare that there is no conflict of interests regarding the publication of this paper.

\section{References}

[1] S. Bellofiore, J. Foutz, C. A. Balanis, and A. S. Spanias, "Smartantenna system for mobile communication networks Part 2: beamforming and network throughput," IEEE Antennas and Propagation Magazine, vol. 44, no. 4, pp. 106-114, 2002.

[2] Y. Kamiya, S. Denno, Y. Mizuguchi, M. Katayama, A. Ogawa, and Y. Karasawa, "Development of an adaptive array based on subband signal processing," in Proceedings of the 5th International Symposium on Antennas, Propagation, and EM Theory (ISAPE '00), pp. 243-246, IEEE, Beijing, China, August 2000.

[3] W. Wiesbeck, RADAR System Engineering, chapter 3, 15th edition, 2008/2009.

[4] http://sanctuaries.noaa.gov/missions/2010coral_west/sonar_ essay.html.
[5] S. Dixit, E. Yanmaz, and O. K. Tonguz, "On the design of selforganized cellular wireless networks," IEEE Communications Magazine, vol. 43, no. 7, pp. 86-93, 2005.

[6] F. Zaman, I. M. Qureshi, A. Naveed, J. A. Khan, and R. M. A. Zahoor, "Amplitude and directional of arrival estimation: comparison between different techniques," Progress in Electromagnetics Research B, vol. 39, pp. 319-335, 2012.

[7] G. Rajshekhar and P. Rastogi, "Multiple signal classification technique for phase estimation from a fringe pattern," Applied Optics, vol. 51, no. 24, pp. 5869-5875, 2012.

[8] M. Hasegawa, H. Kishino, and N. Saitou, "On the maximum likelihood method in molecular phylogenetics," Journal of Molecular Evolution, vol. 32, no. 5, pp. 443-445, 1991.

[9] J. F. Bohme, "Estimation of source parameters by maximum likelihood and nonlinear regression," in Proceedings of the IEEE International Conference on Acoustics, Speech, and Signal Processing (ICASSP '84), pp. 271-274, San Diego, Calif, USA, March 1984.

[10] J. G. Prokis and D. G. Manolokis, Digital Signal Processing, Principles, Algorithms and Applications, Pearson Education, 3rd edition, 1996.

[11] P. Handel, P. Stoica, and T. Soderstrom, "Capon method for doa estimation: accuracy and robustness aspects," in Proceedings of the IEEE Winter Workshop on Nonlinear Digital Signal Processing, pp. P_7.1-P_7.5, Tampere, Finland, January 1993.

[12] P. Todd, T. Hills, and T. Robbins, "Foundation of search: a perspective from computer science," in Cognitive Search: Evolution, Algorithms and Brain, p. 257, MIT Press, Cambridge, Mass, USA, 2012.

[13] M. Melanie, An Introduction to Genetic Algorithms, Massachusetts Institute of Technology, Cambridge, Mass, USA, 5th edition, 1999. 
[14] M. Dorigo and T. Stutzle, "Ant colony optimization: overview and recent advances," in Handbook of Metaheuristics, vol. 146, pp. 227-263, Springer, 2010.

[15] S. Li, N. Bian, Z. Chen, D. You, and Y. He, "A simulation study on some search algorithms for regression test case prioritization," in Proceedings of the 10th International Conference on Quality Software (QSIC '10), pp. 72-81, Zhangjiajie, China, July 2010.

[16] F. Alizadeh, Interior Point Algorithm in Semidefinite Programming with Applications to Combinational Logic, International Computer Science Institute, Berkeley, Calif, USA, 1993.

[17] D. Nettleton and V. Torra, "A comparison of active set method and genetic algorithm approaches for learning weighting vectors in some aggregation operators," International Journal of Intelligent Systems, vol. 16, no. 9, pp. 1069-1083, 2001.

[18] J. L. Morales, J. Nocedal, and Y. Wu, "A sequential quadratic programming algorithm with an additional equality constrained phase," IMA Journal of Numerical Analysis, vol. 32, no. 2, pp. 553-579, 2012.

[19] J. Kennedy and R. Eberhart, "Particle swarm optimization," in Proceedings of the IEEE International Conference on Neural Networks, vol. 4, pp. 1942-1948, Perth, Australia, December 1995.

[20] A. Lazinica, Partical Swarm Optimization, InTech, Rijeka, Croatia, 2009. 


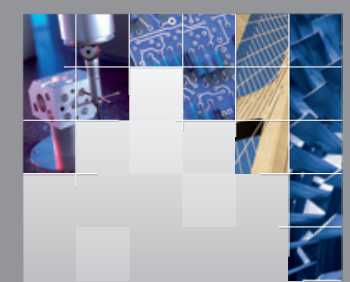

\section{Enfincering}
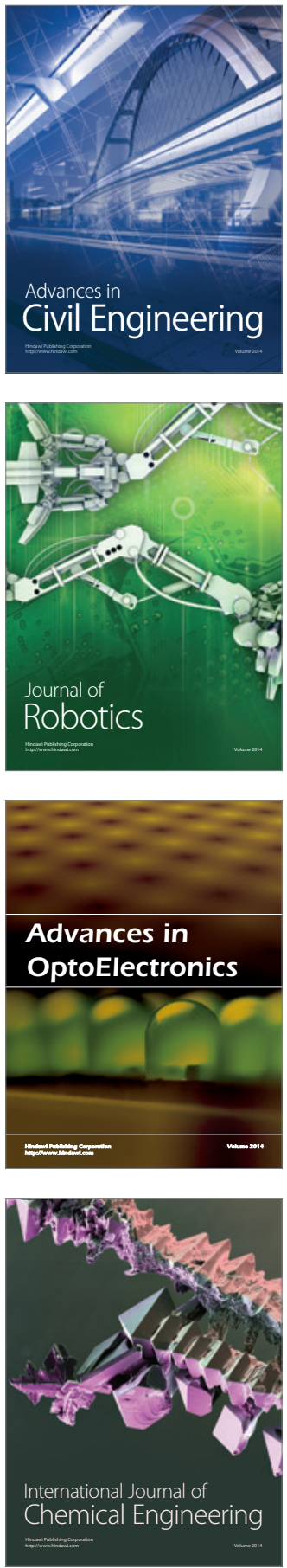

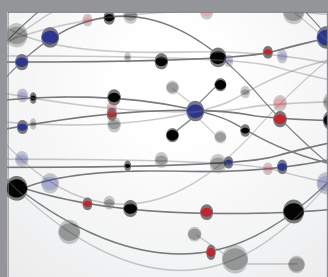

The Scientific World Journal

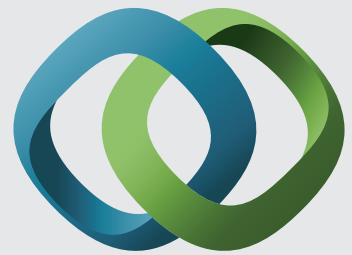

\section{Hindawi}

Submit your manuscripts at

http://www.hindawi.com
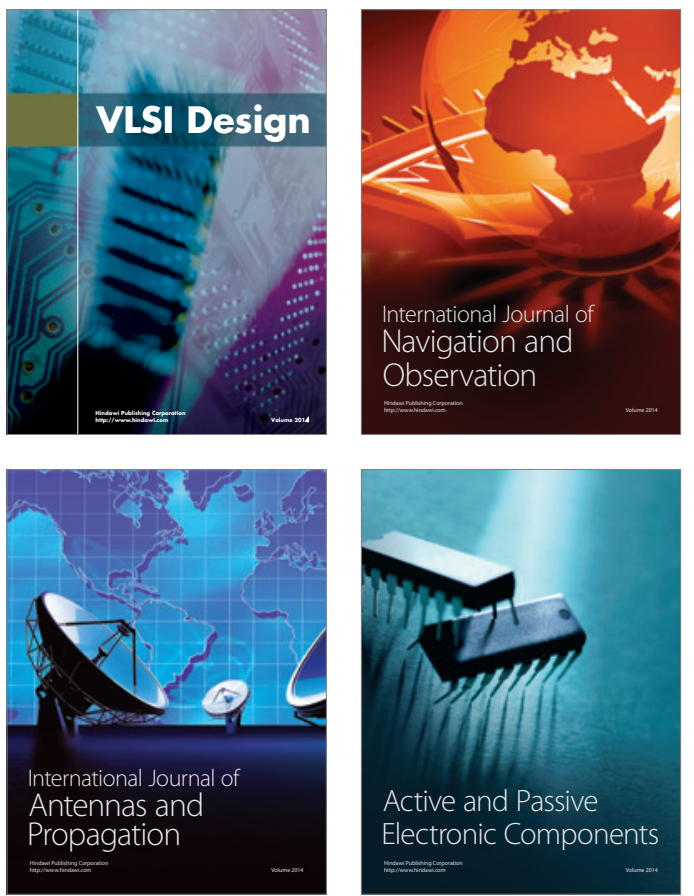
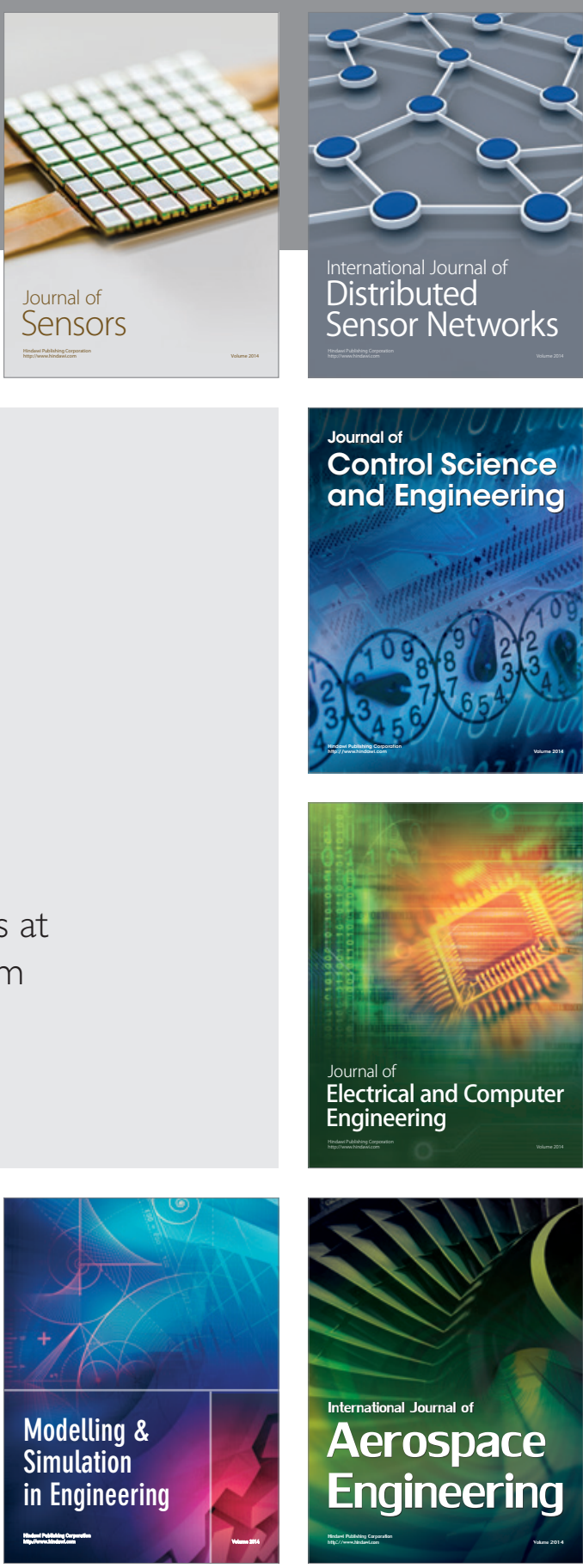

International Journal of

Distributed

Sensor Networks

Journal of

Control Science

and Engineering
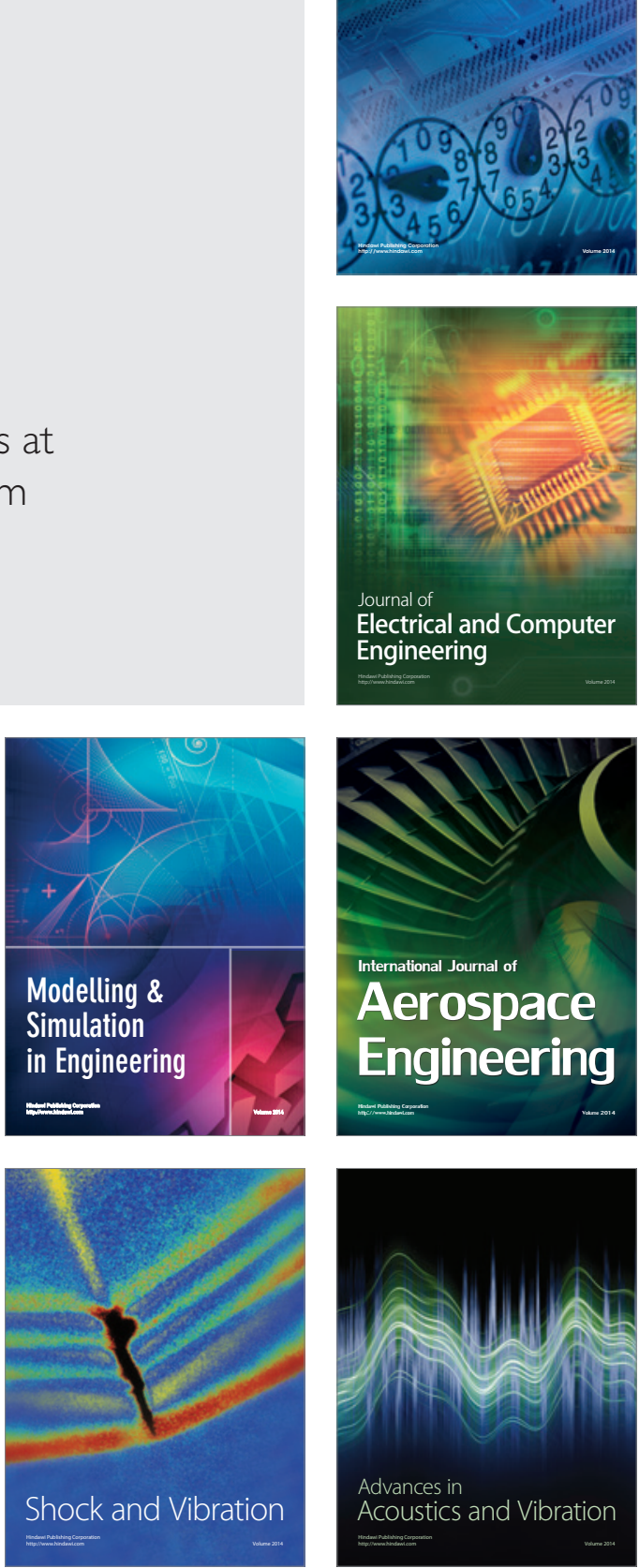